\title{
Cryo-electron microscopy of influenza vaccine nanoparticles indicates full occupancy of displayed epitopes is facilitated by particle design.
}

\author{
John R. Gallagher ${ }^{1}$, Audray K. Harris ${ }^{1}$ \\ 1. Structural Informatics Unit, Laboratory of Infectious Diseases, National Institute of \\ Allergy and Infectious Diseases, National Institutes of Health, Bethesda, MD, USA.
}

Vaccines based upon recombinantly engineered proteins are revolutionizing the process of vaccine design. Presentation of multiple copies of antigen on nanoparticles substantially increases the immune response to vaccination when compared to unitary monovalent counterparts, in part due to multivalent binding sites on immunogens facilitating B-cell receptor activation [1], as well as their size being small enough to permit direct access to the draining lymph and B lymphocyte compartments [2]. Ferritin based nanoparticles have been demonstrated to be a suitable scaffold for antigen presentation [3]. Ferritin based particles displaying antigens from influenza hemagglutinin present 24 such epitopes, but it remains to be demonstrated if all epitopes are accessible on the particle surface, and if the multivalency of the design is fully realized. To address this question, we applied cryo-electron microscopy to reconstruct the nanoparticle in complex with variable region fragments of FABs ( $\mathrm{scFv}$ fragments), and full length FABs, to visualize the sterics of binding.

Ferritin based nanoparticles displaying influenza hemagglutinin stem epitopes from influenza subtype $\mathrm{H} 1$ were expressed and purified from $293 \mathrm{~F}$ cells as described previously [3], and mixed with a twofold excess of F10 scFv fragments. Samples were plunge frozen in liquid ethane and imaged at $300 \mathrm{kV}$ with an FEI Titan Krios and Falcon II camera, and data was collected with FEI EPU. Ferritin nanoparticles in complex with CR6261 FAB were prepared in a 1:1 ratio and imaged in the same manner. CTF correction was performed with CTFFIND3, and image classification and 3D reconstruction was performed with RELION.

We found the ferritin nanoparticle retained octahedral symmetry when bound to $\mathrm{scFv}$ fragments, and a 3D reconstruction of the complex was resolved to $13.8 \AA$. The $\mathrm{scFv}$ fragments were small enough that sterics of binding were unlikely to interfere with full binding to the nanoparticle, and we found $24 \mathrm{scFv}$ fragments could bind to the sides of the antigen spikes without approaching neighboring ligands (Fig. 1). For ligands sized equivalently to the $\mathrm{scFv}$ fragments, all rotational orientations of the antigen spikes could be equally accommodating to full binding. Each epitope is spaced $120 \AA$ apart, and each scFv fragment merely $35 \AA$ in length, allowing ample space for complete binding.

We examined FAB binding because the length of a FAB was large enough that steric occlusion could occur between binding sites. Each FAB is $70 \AA$ in length, thus binding to directly opposed epitopes could be sterically forbidden. A reconstruction of the ferritin nanoparticle in complex with FAB at $17.9 \AA$ resolution could already indicate that the angular orientation of the antigen spikes was permissive to full FAB binding to the nanoparticle (Fig. 2). The angle of FAB binding to antigen spikes was offset $27^{\circ}$ from 
neighboring antigen spikes. Due to symmetry of the complex, neighboring FABs are offset $27^{\circ}$ in the opposite direction, therefore the angular offset sums to $54^{\circ}$ between neighboring antigen spikes. FABs pass between each other on the particle surface, allowing dense and complete binding of FABs on the nanoparticle surface.

Because the effectiveness of nanoparticle vaccines is due largely to their multivalency, it is important to understand if epitopes are sterically occluded by the particle design. We have demonstrated that all epitopes are available for binding interactions, and furthermore that the rigid segment of an antibody, a FAB, is able to occupy all the binding sites on the designed nanoparticle. While future work is needed to determine if Fc domains and bivalent binding may antagonize complete epitope occupancy, our findings indicated that the ferritin nanoparticle scaffold permits presentation of multiple epitopes for multivalent binding while maintaining a size small enough to exploit direct access to the immune system [4].

[1] Maity, P.C., et al., Biochim Biophys Acta, 1853 (2015), p. 830-40.

[2] Smith, D.M., et al., Nat Rev Immunol, 13 (2013), p. 592-605.

[3] Yassine, H.M., et al., Nat Med, 21(2015), p. 1065-70.

[4] The authors acknowledge support from NIH/NIAID, and Vinod Nair and Elizabeth Fischer at the NIAID Rocky Mountain Labs Research Technologies Branch for cryoelectron microscopy data collection.
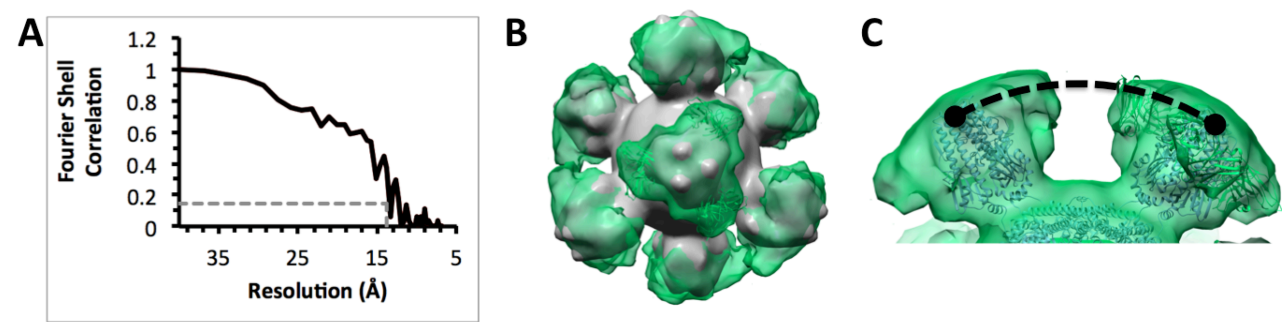

Figure 1. Structure of ferritin nanoparticle displaying hemagglutinin stem epitopes, bound to $24 \mathrm{scFv}$ fragments. (A) FSC curve indicating $13.8 \AA$ resolution. (B) Structure of the complex. (C) Distance between antigen spikes (150 $\AA$ indicated) was permissive to $\mathrm{scFv}$ binding regardless of spike orientation.
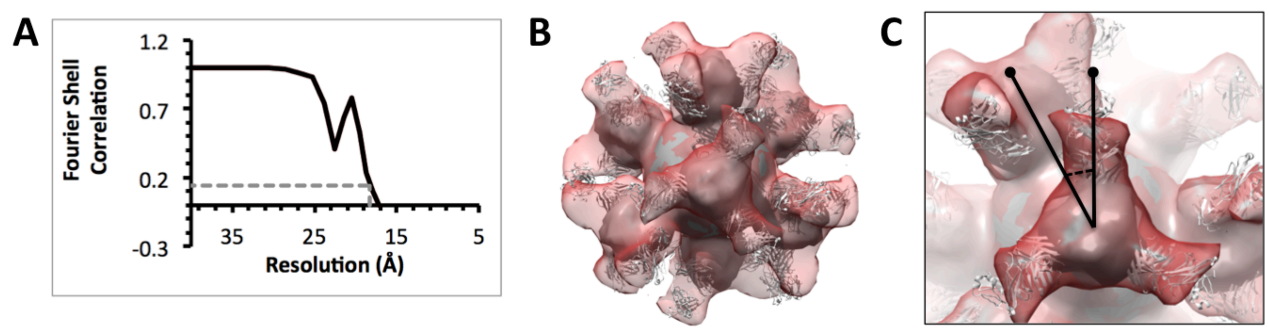

Figure 2. Structure of ferritin nanoparticle displaying hemagglutinin stem epitopes bound to 24 FABs. (A) FSC curve indicating $17.9 \AA$ resolution. (B) Structure of the complex. (C) The indicated offset angle for FAB binding to hemagglutinin spikes is $27^{\circ}$, avoiding steric clash that might have occurred when binding FAB and permitting full occupancy. 